\title{
Summary of Working Group 2
}

\author{
Bruno Autin*, Deborah A. Harris $\uparrow \S$, Steve F. King $\ddagger$, \\ Kevin S. McFarland $* *$ and Osamu Yasuda $\Phi$ \\ * CERN, Geneva, Switzerland \\ ** Department of Physics and Astronomy, University of Rochester, Rochester, \\ NY 14627, USA \\ † Fermi National Accelerator Laboratory, P.O. Box 500, Batavia, IL 60510, USA \\ $\ddagger$ Department of Physics and Astronomy, \\ University of Southampton, Southampton SO17 1BJ, U.K. \\ I Department of Physics, Tokyo Metropolitan University, \\ Minami-Osawa, Hachioji, Tokyo 192-0397, Japan
}

\begin{abstract}
Issues on the physics of, beamlines for, and detectors of neutrino oscillation discussed in Working Group 2 at Nufact'02 are summarized.
\end{abstract}

\section{Introduction}

The way people are thinking about neutrino oscillations and how a neutrino factory fits into a program of measurements has changed dramatically between previous NuFact workshops and this one. Although we still consider a neutrino factory the ultimate facility to do precision oscillation measurements, we are becoming more aware of what one can learn with conventional neutrino beams. Understanding how much of the possible parameter space one can explore with conventional beams, and how these experiments might complement neutrino factory measurements has kept many phenomenologists busy this past year. The oscillation working group also has included for the first time discussions on different proposals for these super conventional neutrino beams, as well as a new idea for making a pure $\nu_{e}$ or $\bar{\nu}_{e}$ beam, which can be done by accelerating radioactive ions which undergo beta decay. With these new techniques for measurements, new issues become relevant: cross sections, beam systematics, and backgrounds, the three things that were so straightforward to tackle in the case of neutrino factory experiments.

While the experimental picture is changing, so too is the theoretical motivation for studying neutrino oscillations. With the concept of extra dimensions becoming more fleshed out in the past year, neutrinos have surfaced as an example of particles which have significant activity outside the brane we live on. Furthermore, given that the SNO Experiment has confirmed solar oscillations, and in particular, confirmed that the mixing angle describing solar neutrino oscillations is large, the prospect of seeing leptonic CP violation in the laboratory is still alive. The see-saw mechanism remains an attractive candidate for accounting for the smallness of neutrino mass and can easily accommodate the observed large solar angle. Furthermore the see-saw mechanism allows the possibility that baryon asymmetry is generated in the early

$\S$ Corresponding author (dharris@fnal.gov) 
universe, but only if $\mathrm{CP}$ is violated in the lepton sector. In the past year there has been much theoretical work trying to make the link between the $\mathrm{CP}$ violation required by baryon asymmetry and that potentially observable in low energy neutrino experiments.

In this summary we try to give an overview of what the issues are in both understanding the origin of neutrino oscillations, how to go from experimental measurements to oscillation parameters, and finally how to make the experimental measurements themselves.

\section{Theory}

Perhaps the zeroth order question is whether we are actually seeing atmospheric and solar neutrino oscillations, or some other non-standard effect? Valle [1] showed that while the case for atmospheric oscillations looks convincing, there are alternatives to solar oscillations which fit the data well, such as spin flavor precession or flavor changing neutrino interactions. However the magnetic moments and fields, or flavor changing couplings do not appear very natural, and an oscillation signal from KamLAND would rule out such interpretations.

Assuming we are seeing oscillations, and hence neutrino mass, then, ignoring LSND for the moment (discussed later), the atmospheric and solar data imply two possible patterns of neutrino mass, distinguished by the sign of $\Delta m_{32}^{2}$, together with two large mixing angles and one small mixing angle (also discussed later). From a theoretical point of view the challenge is to account for such a neutrino spectrum. The first point to make is that neutrino masses are zero in the Standard Model, so neutrino oscillations are the first solid evidence for new physics beyond the Standard Model. Three possible ways to extend the Standard Model in order to account for the neutrino mass spectrum above were discussed in this working group, namely the see-saw mechanism, extra dimensions, and R-parity violating supersymmetry.

In his plenary talk Ross [2] reviewed the see-saw mechanism in which super-heavy right-handed Majorana neutrinos are introduced into the Standard Model, which induce light effective left-handed Majorana neutrino masses according to the formula $m_{\nu} \sim m_{f}^{2} / M$ where $m_{f}$ represents a typical quark or charged lepton mass and $M$ is the heavy right-handed neutrino mass scale. He showed that if one of the right-handed neutrinos plays the dominant role in the see-saw mechanism then a mass hierarchy and large mixing angles may naturally emerge.

Blazek [3] applied the see-saw mechanism to GUT models such as $S O(10)$ where three families of right-handed neutrinos with Majorana masses not far below the GUT scale are predicted. GUT models require supersymmetry (SUSY) in order to allow the gauge couplings to meet, and also to stabilize the hierarchy. One of the consequences of having a see-saw mechanism with SUSY is lepton flavor violation (LFV) which was discussed extensively in this working group. Blazek 3 showed that sizable branching ratios for $\tau \rightarrow \mu \gamma$ are expected. Shimizu [4] confirmed this and presented results for $\mu \rightarrow e \gamma$ which also showed large branching ratios. Shimoyama [5] showed that in a simplified model where right-handed neutrinos have universal masses the standard SUSY model implies branching ratios for $\mu \rightarrow e \gamma$ which exceed the experimental limit. Finally Koike [6] studied the LFV process $\mu+N_{Z}^{A} \rightarrow e+N_{Z}^{A}$ and concluded that $Z \sim 30-60$ represents the optimal range for experimental searches. He also showed how it may be possible to distinguish models of new physics by measurements on several different kinds of nuclei. 
The see-saw mechanism also opens up the possibility of generating the baryon asymmetry of the universe via leptogenesis. The idea due to Fukugita and Yanagida [7] is that in the early universe the super-heavy lepton number violating Majorana right-handed neutrinos would have been produced, and, if CP is violated, could then decay out of equilibrium giving a lepton asymmetry which is subsequently converted into baryon asymmetry via electroweak sphalerons. The possible link between the $\mathrm{CP}$ violating phases necessary for leptogenesis and the $\mathrm{CP}$ violating phases measurable by low energy neutrino physics was also explored in this working group. Working in a simple framework of two right-handed neutrinos, Morozumi [8] discussed the possible correlation between leptogenesis and low energy phases. Ibarra 9] discussed the case of three right-handed neutrinos, and showed that although in general there is no direct link, the low energy observable phases may contribute to leptogenesis over much of the parameter space.

Neutrino masses in the framework of extra dimensions were considered by Shafi [10], who specialized to the case of a 5D standard model with warped geometry. The basic idea is that the Standard Model fermions reside in the 5D bulk, and the quark and lepton mass hierarchies can be interpreted in a geometrical way, with the higgs field localized at the $\mathrm{TeV}$ brane, and the fermion masses being in direct proportion to the overlap of their wavefunctions with the higgs field. In such a set-up the seesaw mechanism cannot be implemented because of the warped geometry, however the desired Majorana masses may arise from non-renormalizable operators, and the masses again depend on the position of the left-handed leptons in the bulk.

Neutrino masses in R-parity violating (RPV) SUSY were discussed by Valle [1]. In the simplest bilinear RPV scheme one adds an explicit mass term coupling lepton doublets to a Higgsino doublet. The left-handed neutrinos are then mixed in the neutralino mass matrix, resulting in a single neutrino Majorana mass term. The other neutrino masses and mixings are generated by loop corrections, depending on the RPV SUSY parameters. This results in a testable relation between neutrino masses and RPV SUSY physics at colliders.

\section{Phenomenology}

\subsection{Three flavor mixing}

The observation of atmospheric neutrinos (See, e.g., Ref.[1]) and solar neutrinos (See, e.g., Ref.[12]) implies that not only are neutrinos massive, but that the mass eigenstates $\left(\nu_{1}, \nu_{2}, \nu_{3}\right)$ with mass $\left(m_{1}, m_{2}, m_{3}\right)$ are not identical to the flavor eigenstates $\left(\nu_{e}, \nu_{\mu}, \nu_{\tau}\right)$. The unitary matrix which translates from the mass to the flavor basis is usually parameterized by three mixing angles $\left(\theta_{12}, \theta_{23}, \theta_{13}\right)$, and a CP violating phase $(\delta)$, and is often referred to as the MNSP [13, 14] matrix, after Maki, Nakagawa, Sakata, and Pontecorvo. The atmospheric data largely constrain $\left(\left|\Delta m_{32}^{2}\right|\right.$, $\left.\theta_{23}\right)$ and the solar data constrain $\left(\Delta m_{21}^{2}, \theta_{12}\right)$ in this three flavor framework of neutrino oscillations [15], where $\Delta m_{i j}^{2} \equiv m_{i}^{2}-m_{j}^{2}$. The constraint from the CHOOZ result [16] implies $\sin ^{2} 2 \theta_{13}<0.1$. So in vacuum the MNSP matrix looks roughly like

$$
U_{M N S P} \simeq\left(\begin{array}{ccc}
c_{\odot} & s_{\odot} & \epsilon \\
-s_{\odot} / \sqrt{2} & c_{\odot} / \sqrt{2} & 1 / \sqrt{2} \\
s_{\odot} / \sqrt{2} & -c_{\odot} / \sqrt{2} & 1 / \sqrt{2}
\end{array}\right),
$$

where we have used $\theta_{23} \simeq \pi / 4$ and $|\epsilon| \ll 1$, and $c_{\odot}=\cos \theta_{12}$, and $s_{\odot}=\sin \theta_{12}$. 
Choubey [17] performed model independent and model dependent analyses of solar neutrino data including the neutral current event rate from SNO [18. She concluded that the Large Mixing Angle solution gives the best fit $\left(\sin ^{2} 2 \theta_{12} \simeq 0.8\right)$ and the LOW solution is allowed only at the $3 \sigma$ level. Valle 1 also gave results on his solar neutrino analyses and his results agree with those in [17. While whether the LMA solution is the correct one or not will be soon confirmed by the KamLAND experiment, it should be noticed that the goodness of fit (GOF) for each of the other solar solutions (LOW, Quasi Vacuum Oscillation, Small Mixing Angle solutions) is still acceptable. Namely, according to [17, we have $\chi^{2}(\mathrm{LMA})=40.57$, $\chi^{2}(\mathrm{LOW})=50.62, \chi^{2}(\mathrm{QVO})=56.11$, and $\chi^{2}(\mathrm{SMA})=70.97$ for 45 degrees of freedom. This implies that LMA is $65.99 \%(0.44 \sigma \mathrm{CL})$ likely, LOW is $26.14 \%(1.12 \sigma \mathrm{CL})$ likely, QVO is $12.39 \%(1.54 \sigma \mathrm{CL})$ likely, and SMA is $0.81 \%(2.65 \sigma \mathrm{CL})$ likely. As far as GOF is concerned, none of these solutions is excluded from a conservative point of view.\| They are disfavored when they are compared with the LMA solution, i.e., $\Delta \chi^{2}=$ $\chi^{2}(Q V O)-\chi^{2}(\mathrm{LMA})=15.54(3.53 \sigma \mathrm{CL}$ for d.o.f. $=2), \Delta \chi^{2}=\chi^{2}(S M A)-\chi^{2}(\mathrm{LMA})$ $=30.40(5.16 \sigma \mathrm{CL}$ for d.o.f. $=2)$. On the other hand, 20 argues that GOF is a necessary but not sufficient criterion for a model to survive. 20] claims that the SMA solution is rejected because it creates strong tension between not only total rate versus spectrum data, but also between SNO data versus all the others, while the LOW and QVO solutions are still acceptable because the tension is much smaller for them than that for SMA.

With the mass hierarchy $\left|\Delta m_{21}^{2}\right| \ll\left|\Delta m_{32}^{2}\right|$ there are two possible mass patterns, depending on whether $\Delta m_{32}^{2}$ is positive (normal hierarchy: $m_{1}^{2}, m_{2}^{2} \ll m_{3}^{2}$ ) or negative (inverse hierarchy: $\left.m_{3}^{2} \ll m_{1}^{2}, m_{2}^{2}\right)$. The quantities one has to determine are $\theta_{13}$, the sign of $\Delta m_{32}^{2}$ and the $\mathrm{CP}$ phase $\delta$. To discuss $\mathrm{CP}$ violation in long baseline experiments, it is helpful to have the exact analytical expressions for the oscillation probabilities in matter. Kimura [21] gave the expressions in a nice way. The point of his work is that the quantity $\tilde{X}_{j}^{\alpha \dot{\beta}} \equiv \tilde{U}_{\alpha j} \tilde{U}_{\beta j}^{*}(\alpha, \beta=e, \mu, \tau$ are the flavor indices, $j=1,2,3$ is the index of the mass eigen states, and no sum on $j$ is implied) in matter with constant density can be expressed linearly in the quantity $X_{j}^{\alpha \beta} \equiv U_{\alpha j} U_{\beta j}^{*}$ in vacuum with some coefficients which are functions of the eigenvalues $\tilde{E}_{j}$ of the $3 \times 3$ matrix $U \operatorname{diag}\left(E_{1}, E_{2}, E_{3}\right) U^{-1}+\operatorname{diag}\left(\sqrt{2} G_{F} N_{e}, 0,0\right)$, where $E_{j} \equiv \sqrt{p^{2}+m_{j}^{2}}$. His results are more useful than previous works (e.g., 22]) since the expression does not use a particular parametrization for $U_{\alpha j}$ and it is relatively easy to see the condition in which enhancement of the oscillation probability occurs. Once we know the form for $\tilde{X}_{j}^{\alpha \beta}$, we can write down the the oscillation probability as

$$
\begin{aligned}
P\left(\nu_{\alpha} \rightarrow \nu_{\beta}\right)=\delta_{\alpha \beta} & -4 \sum_{j<k} \Re\left(\tilde{X}_{j}^{\alpha \beta} \tilde{X}_{k}^{\alpha \beta *}\right) \sin ^{2}\left(\frac{\Delta \tilde{E}_{j k} L}{2}\right) \\
& +2 \sum_{j<k} \Im\left(\tilde{X}_{j}^{\alpha \beta} \tilde{X}_{k}^{\alpha \beta *}\right) \sin \left(\Delta \tilde{E}_{j k} L\right),
\end{aligned}
$$

where $\Delta \tilde{E}_{j k} \equiv \tilde{E}_{j}-\tilde{E}_{k}$.

\| The criterion in the 1996 version of Particle Data Book (sect. 28.5 in [19]) is that $4 \sigma$ or $5 \sigma$ is necessary to exclude a hypothesis. 


\subsection{Parameter degeneracy}

Naively one might assume that with two general unknowns, $\left(\theta_{13}, \delta\right)$, and one discrete unknown, (the sign of $\Delta m_{32}^{2}$ ), and two measurements of the oscillation probability at a neutrino factory (i.e. $\nu_{e} \rightarrow \nu_{\mu}$ and $\bar{\nu}_{e} \rightarrow \bar{\nu}_{\mu}$ ) it would be straightforward to extract the mixing parameters. However, since the work 23], it has been realized that there is in general more than one solution for the set of oscillation parameters, even if we know both $P\left(\nu_{\mu} \rightarrow \nu_{e}\right)$ and $P\left(\bar{\nu}_{\mu} \rightarrow \bar{\nu}_{e}\right)$. Reference 23] shows that there are intrinsically two sets of solutions $\left(\theta_{13}, \delta\right)$ and $\left(\theta_{13}^{\prime}, \delta^{\prime}\right)$. Reference 24] describes the degeneracy under $\Delta m_{32}^{2} \leftrightarrow-\Delta m_{32}^{2}$, and Reference [25, 26] found the ambiguity under $\theta_{23} \leftrightarrow \pi / 2-\theta_{23}$. Thus there is an eight-fold degeneracy [26], and the resolution of this degeneracy was one of the main subjects at Nufact'02. The degeneracy in $\theta_{23} \leftrightarrow \pi / 2-\theta_{23}$ is difficult to resolve as long as we look at only channels $\nu_{\mu} \leftrightarrow \nu_{e}, \bar{\nu}_{\mu} \leftrightarrow \bar{\nu}_{e}$ and $\nu_{\mu} \rightarrow \nu_{\mu}$, since the first two create the degeneracy while the third gives the information only on $\sin ^{2} 2 \theta_{23}$. As was pointed out in 26, the best way is probably to look at the channel $\nu_{e} \rightarrow \nu_{\tau}$ whose oscillation probability in vacuum is given by $\cos ^{2} \theta_{23} \sin ^{2} 2 \theta_{13} \sin \left(\Delta m_{32}^{2} L / 4 E\right)$, which is compared with $P\left(\nu_{e} \rightarrow \nu_{\mu}\right)=\sin ^{2} \theta_{23} \sin ^{2} 2 \theta_{13} \sin \left(\Delta m_{32}^{2} L / 4 E\right)$.

To determine $\theta_{13}$, Minakata 24 proposed to tune the neutrino energy at the oscillation maximum $\left(\Delta m_{32}^{2} L / 4 E=\pi / 2\right)$ so that the ellipse in the $P\left(\nu_{\mu} \rightarrow \nu_{e}\right)-$ $P\left(\bar{\nu}_{\mu} \rightarrow \bar{\nu}_{e}\right)$ plane that results from letting $\delta$ run from 0 to $2 \pi$ collapses to a line. $\uparrow$ Then the ambiguity in $\theta_{13}$ is reduced.

Whisnant 28 discussed systematically whether or not a set of measurements in neutrino oscillation appearance experiments can completely resolve the ambiguities. He showed that experiments with neutrinos and antineutrinos at two different energies, which give the four independent measurements, can in principle resolve all of the parameter degeneracies if $\sin ^{2} 2 \theta_{13} \geq 0.002$.

Mena [29] showed that by combining a superbeam and a neutrino factory, it is possible to reduce the intrinsic, $\operatorname{sign}\left(\Delta m_{13}^{2}\right)$ and $\theta_{23}$ degeneracies. She pointed out that the short neutrino factory baseline (e.g. $L=732 \mathrm{~km}$ ) becomes an interesting distance to combine with a superbeam experiment for values of $\theta_{13}$ near its present limit. For smaller $\theta_{13}$, an intermediate neutrino factory baseline of $O(3000 \mathrm{~km})$ is still required.

Meloni [30] showed how the $\nu_{e} \rightarrow \nu_{\mu}$ ("golden") and $\nu_{e} \rightarrow \nu_{\tau}$ ("silver") transitions observed at an OPERA-like 2 Kton lead-emulsion detector at $L=732 \mathrm{Km}$, in combination with the $\nu_{e} \rightarrow \nu_{\mu}$ transitions observed at a 40 Kton magnetized iron detector with a baseline of $L=3000 \mathrm{Km}$, strongly reduce the intrinsic $\left(\theta_{13}, \delta\right)$ ambiguity. He also showed how a moderate increase in the OPERA-like detector mass (4 Kton instead of 2 Kton) completely eliminates the degenerate regions even for small values of $\theta_{13}$.

Huber 31 compared the physics potential of planned superbeams with the one of neutrino factories. He showed that JHF-SuperK $(0.75 \mathrm{MW}, 110 \mathrm{Kt} \cdot \mathrm{yr})$, JHF-HyperK (4MW, 8Mt.yr) and NuFact-I (0.75MW, 50Kt·yr), NuFact-II (4MW, 400Kt.yr) can explore $\sin ^{2} 2 \theta_{13}$ down to $\simeq 2 \cdot 10^{-2}, \simeq 10^{-3}, \simeq 5 \cdot 10^{-4}$, respectively, while only JHFHyperK and NuFact-II can probe the CP phase for $\sin ^{2} 2 \theta_{13} \geq$ several $\times 10^{-3}, 10^{-4}$, respectively.

A previous study showed that two simultaneous baselines for a neutrino factory

could be used to resolve degeneracies 23 where something like a combination of $3000 \mathrm{~km}$ plus either $700 \mathrm{~km}$ or $7000 \mathrm{~km}$ baselines might be optimal. For one neutrino

I The idea to tune the neutrino energy at the oscillation maximum was given earlier in [27. 
factory to access two baselines, a triangular or bow-tie ring would be necessary. Depending on what the first generation of neutrino superbeams measures, the argument for making two simultaneous baselines may be less compelling.

\subsection{Matter effect}

In very long baseline experiments, it is important to estimate the magnitude of the matter effect. Unlike in shorter or medium long baseline experiments, in which neutrinos go through only the crust or the upper mantle of the Earth, a constant density approximation is not good in very long baseline experiments.

By using the method of a Fourier series expansion of the matter profile, Ota 32 showed that neglecting the matter profile effect leads to an extra uncertainty $(\sim 5 \%)$ on the average density for $5,000 \mathrm{~km} \lesssim L \lesssim 10,000 \mathrm{~km}$. He proposed to treat the first coefficient in the Fourier series as a fitting parameter.

Winter 33 compared three different methods (single perturbation, random fluctuations, and the measured mean) to evaluate uncertainties in the Earth's matter density profile. He gave advantages and disadvantages for each method and he concluded that matter density uncertainties probably would not be the bottleneck of the statistical analysis of a planned experiment, while he claimed that somewhat more effort should be spent on improving the results from geophysics.

\subsection{Exotic scenarios}

Although the LSND result [34 is not yet confirmed, we can still try to explain the LSND result in at least three different ways. The first way is to assume a fourth mass eigenstate, which implies one sterile neutrino (with a $(2+2)$ or $(3+1)$ mass scheme, depending on the mass pattern). The LSND signature is then simply the result of three independent mass squared differences. Valle 1 gave the results of his recent analyses on the solar and atmospheric neutrino data for the $(2+2)$-scheme, and it is strongly disfavored. The $(3+1)$-scheme is allowed only at $99 \% \mathrm{CL}$. A second possibility is to give up CPT invariance [35] and to take full advantage of the difference between neutrinos and antineutrinos. This possibility would be excluded if KamLAND observes the deficit. The third idea [36] is to assume a lepton number violating interaction $\mu^{+} \rightarrow e^{+}+\bar{\nu}_{e}+\bar{\nu}_{\alpha}(\alpha=e, \mu, \tau)$. Interestingly, this scenario predicts that the MiniBooNE experiment will see no signal. There are other exotic scenarios which can be tested at neutrino factories.

Wong [37. considered the situation where the equivalence principle is violated in neutrino oscillations with three flavors. The $3 \times 3$ matrix which describes the propagation of neutrinos is

$$
H=U \operatorname{diag}\left(E_{1}, E_{2}, E_{3}\right) U^{-1}+\operatorname{diag}\left(\sqrt{2} G_{F} N_{e}, 0,0\right)+2 E|\phi| U_{G} \operatorname{diag}\left(\gamma_{1}, \gamma_{2}, \gamma_{3}\right) U_{G}^{-1},
$$

where $\gamma_{j}$ is the relative gravitational coupling constants, Violation of the Equivalence Principle (VEP) implies $\gamma_{j} \neq 1, U_{G}$ stands for the mixing matrix between the gravitational and flavor eigenstates, and $\phi$ is the gravitational potential. Assuming $|\Delta \gamma| \equiv\left|\gamma_{3}-\gamma_{2}\right| \gg\left|\gamma_{2}-\gamma_{1}\right|$ for simplicity, she gets the following ratio of $\mathrm{T}$ violation $\Delta \tilde{P}_{T}$ with VEP to that $\Delta P_{T}$ without VEP

$$
\frac{\Delta \tilde{P}_{T}}{\Delta P_{T}}=1-\frac{4 E^{2}}{\Delta m_{21}^{2}} \frac{\sin 2 \theta_{\mathrm{atm}}}{\sin 2 \theta_{\odot}}|\phi| \Delta \gamma \sin 2 \varphi,
$$


where $\varphi$ is the mixing angle between the first and the third eigenstates in $U_{G}$ and other mixings in $U_{G}$ are assumed to be zero for simplicity. She showed that $\mathrm{T}$ violation $\Delta \tilde{P}_{T}$ with VEP is deviated from $\Delta P_{T}$ without VEP if the violation $|\Delta \gamma||\phi|$ is large and therefore may be measured in long baseline experiments.

Sato 38 discussed the measurement of new physics which is described by an interaction such as $g\left(\bar{e} \gamma_{\lambda} \mu\right)\left(\bar{\nu}_{\mu} \gamma^{\lambda} \nu_{\beta}\right)(\beta \neq e)$ in long baseline neutrino oscillation experiments. Through the neutrino oscillation, the probability to detect the new physics effects such as flavor violation is enhanced by the interference with the weak interaction. Assuming a neutrino factory and an upgraded conventional beam, he examined the possibility to observe new physics numerically and pointed out that it is possible to search for new interactions using some channels, for example $\nu_{\mu} \rightarrow \nu_{\mu}$, in these experiments. He also showed that there is a chance to observe a flavor-changing effect in $\tau$ appearance experiments such as ICARUS and OPERA.

Campanelli 39 discussed a new flavor changing interaction which gives the contribution $\left(\epsilon_{\alpha \beta}\right)(\alpha \beta=e, \mu, \tau)$ to the $3 \times 3$ matrix in the flavor basis. He showed that the new physics effect becomes important in the channels $\nu_{e} \rightarrow \nu_{\alpha}(\alpha=\mu, \tau)$ at sufficiently high neutrino energies, since the contribution from the conventional physics is suppressed by $\theta_{13}$ and $1 / E_{\nu}$. Therefore it may be possible to observe the new physics effect particularly by looking at the energy spectrum which would be different in the presence of new physics.

\section{Oscilation Measurements}

It is clear from the discussion above that to measure the oscillation parameters and to probe exotic scenarios, we will need more than one measurement of oscillations to understand phenomena visible at the atmospheric $\delta_{m}^{2}$. Furthermore, there is much than can be learned at high intensity, conventional (that is, meson decay) beams. If a signal is seen in a conventional beam, that information may still be an important constraint even in the advent of a neutrino factorybecause of the different energies of the beams. The challenge with doing any of the measurements described above in conventional neutrino beams is that the $\nu_{\mu} \rightarrow \nu_{e}$ transition probability is limited by the CHOOZ non-observation of $\nu_{\mu}$ disappearance to be small, less than $5 \%$ in the absence of enhancement from $\mathrm{CP}$ violation or matter effects. Intrinsic $\nu_{e}$ backgrounds in conventional beams at the per cent level, due primarily to the decay chain $\pi^{+} \rightarrow \nu_{\mu} \mu^{+}, \mu^{+} \rightarrow e^{+} \nu_{e} \bar{\nu}_{\mu}$, and significant background processes present if neutrino energies are above a few hundred $\mathrm{MeV}$ from neutral current events mimicking electron signals will make these measurements difficult.

For a given detector mass, one gets the best sensitivity for seeing a non-zero value of the muon to electron neutrino transition probability by placing an experiment near or slightly below the first oscillation maximum $\left(1.27 \Delta m^{2} L / E \approx \pi / 2\right)$. In order to see evidence for matter effects, and thereby deduce the mass hierarchy, however, one needs to go to baselines on the order of $1000 \mathrm{~km}$, and therefore use neutrino energies well above $1 \mathrm{GeV}$. Finally, to look for $\mathrm{CP}$ violation, one might still run at the first oscillation peak, as described by Minakata 24, but it has also been argued that one should consider running an experiment at the second or third oscillation maximum [40], since the CP-violating contribution to the transition grows with $L / E$.

To get around the problem of backgrounds, several different strategies have been proposed. For example, the JHF to SuperK, the NUMI Off-axis, and the CNGT proposals all take advantage of the two-body decay kinematics of the pion, and put 
the far detector slightly off the main axis of the neutrino beamline 41, 42, 46. This trick, first suggested in BNL proposal E889 [43, allows one to maximize the $\nu_{\mu}$ flux in a very narrow energy region, reduces the flux at higher neutrino energies, and does not alter the flux from three-body decays, which are the source of intrinsic $\nu_{e}$ 's in the beam. This results in an improved signal to background ratio at the peak beam energy since the background from high $E_{\nu}$ neutral current interactions that fake electron charged current events at this peak energy is reduced. However, since the $\nu_{\mu}$ spectrum is significantly narrower than the shape of the appearance probability, one suffers from statistics. This must be mitigated by the use of massive detectors and very high proton beam power.

Another way to reduce the effect of backgrounds is to consider a distance of thousands of kilometers where the matter effects will significantly amplify either the neutrino or the antineutrino probability, so that even for a small value of $\sin ^{2} 2 \theta_{13}$ a large probability would be expected. This is the strategy of the Brookhaven to NUSL proposal, as described by S.Kahn [4]. However, to go such large distances, even with a wide band beam, requires a "megaton class" detector. By combining a large distance and a broad band neutrino energy spectrum, this proposal can see not only the first but the second oscillation maximum, and as such can have more constraints on the oscillation parameters.

A third way to reduce the backgrounds inherent in a conventional beam is to go to neutrino energies of a few hundred $\mathrm{MeV}$ and to construct only a short decay tunnel so that few muons decay to produce $\nu_{e}$ 's, as described by M.Mezzetto. The intrinsic $\nu_{e}$ contamination of the proposed CERN SPL beam is at the parts per mil level, and at this neutrino energy the few neutral pions that do get produced are easy to distinguish from electrons since there is a large angle between the two decay photons. However in the few hundred $\mathrm{MeV}$ energy range, the cross-section varies quadratically with energy, suppressing the rate, so again a megaton-class detector must be used.

Finally, a novel technique for creating a beam with extremely low backgrounds is to accelerate a beam of radioactive ions in a long storage ring, and let them decay to electron neutrinos. The intrinsic backgrounds here are just as low as that of a neutrino factory, and in fact the beam is really only $\nu_{e}$ or $\bar{\nu}_{e}$ depending on the ion in the ring, with contaminations from any other flavor at less than the $10^{-4}$ level. The resulting neutrino beam would be at a very low neutrino energy, and a water Черенков ${ }^{+}$detector located in the Frejus tunnel could be used for both this "beta beam" concept and the CERN SPL neutrino beam. This concept was presented in the plenary session by P. Zucchelli and in a parallel session by M. Mezzetto.

Table 4 gives a summary of the different neutrino experiments that are being considered worldwide, and the different strategies being adopted. The experiments listed below are at very different stages of completion, as well: the JHF to SuperKamiokande uses an existing 50 kTon detector, but will require a new beamline to be built at the Japanese Hadron Facility. The beamline is being designed not only to handle both a first stage experiment with a 1MW proton source, but also an upgraded stage with a 4MW proton source, and a megaton-class water Черенков detector [45]. The NUMI Off-axis experiment would use the (almost) existing NUMI beamline, but a new $\sim 30$ kTon detector would need to be built somewhere in northern Minnesota or perhaps even in Canada [46]. The CNGT project would require instrumenting a

+ Here we use the correct Cyrillic spelling and not the often seen used ethnically inaccurate version with the Čech accent 
Summary of Working Group 2

9

\begin{tabular}{|c|c|c|c|c|c|c|c|}
\hline $\begin{array}{l}\text { Program } \\
\text { Name }\end{array}$ & $\begin{array}{c}<E_{\nu}> \\
\mathrm{GeV}\end{array}$ & $\begin{array}{c}\mathrm{L} \\
\mathrm{km}\end{array}$ & $\begin{array}{l}\text { Proton } \\
\text { Power } \\
\text { (MW) }\end{array}$ & $\begin{array}{c}\text { Detector } \\
\text { Mass } \\
\text { (kton) }\end{array}$ & $\begin{array}{c}\text { Wide or } \\
\text { Narrow } \\
\text { Band }\end{array}$ & $\begin{array}{c}\text { Which } \\
\max \\
?\end{array}$ & $\begin{array}{c}\text { Matter } \\
\text { Effect } \\
\text { Size }\end{array}$ \\
\hline JHF to $\mathrm{SK}^{a}$ & 0.77 & 295 & 0.75 & 22.5 & Narrow & 1 & tiny \\
\hline NUMI OA ${ }^{b}$ & 2 & $\simeq 730$ & 0.4 & 20 & Narrow & 1 & big \\
\hline BNL to $\mathrm{NY}^{c}$ & 1 & & 1 & 500 & Wide & 1 & tiny \\
\hline BNL to NUSL $^{c}$ & 1 & 2600 & 1 & 500 & Wide & 1 & huge \\
\hline SJHF to HK ${ }^{a}$ & 0.77 & 295 & 4 & 1000 & Narrow & 1 & tiny \\
\hline SNUMI OA ${ }^{b}$ & 2 & $\simeq 1000 ?$ & 2 & 100 & Narrow & 1 & bigger \\
\hline $\mathrm{SPL}^{d}$ & 0.27 & 130 & 4 & 500 & Wide & $<1$ & none \\
\hline $\mathrm{CNGT}^{e}$ & 0.8 & 1200 & $\sim 0.5^{e e}$ & 1000 & Narrow & 1 & big \\
\hline$\beta$ beams $^{d}$ & 0.13 & 130 & $\mathrm{n} / \mathrm{a}$ & 500 & wide & 1 & none \\
\hline$\nu$ Factory $^{f}$ & $10-30$ & 3000 & 4 & 50 & Wide & $<1$ & huge \\
\hline
\end{tabular}

Table 1. Vital characteristics of future possible neutrino experiments. ee Proton power of CNGT assumed to be approximately that of CNGS. Speakers in this workshop were: ${ }^{a} \mathrm{~A}$. Ichikawa, ${ }^{b} \mathrm{~S}$. Kopp, ${ }^{c} \mathrm{~S}$. Kahn, ${ }^{d} \mathrm{M}$. Mezzetto, ${ }^{e} \mathrm{~B}$. Autin, ${ }^{f}$ S. Geer.

large body of water, perhaps in the gulf of Taranto for a detector, and the CNGS beamline would have to be tuned at a lower energy which would preclude measuring $\tau$ appearance [42] at Gran Sasso. Finally, the other programs (CERN SPL and the BNL proposal) require both new beamlines and new detectors [44, 47].

\subsection{Far Detector Strategies}

For most of the proposals listed in table 4 the detector of choice is a water Черенков device-either the SuperKamiokande detector, or a new larger version, which would also presumably be instrumented well enough to search for proton decay and supernovae neutrinos. At energies at or below $1 \mathrm{GeV}$, the SuperKamiokande detector is able to keep detector misidentification at a level comparable to or below the intrinsic electron neutrino backgrounds in the JHF off-axis beamline.

For a neutrino factory, the energies are $10 \mathrm{~s}$ of $\mathrm{GeV}$, and because charge identification is required, there is an appropriate and proven candidate detector technology, a steel-scintillator detector like the one being built for the MINOS experiment [48]. For the $\nu_{e} \rightarrow \nu_{\tau}$ measurements that were described by Sato and Meloni, an OPERA-like detector, even one that was twice the mass of the current design, could provide very interesting measurements.

A detector technology which in principle could work for any of the conventional beamlines, is a liquid argon TPC, such as that being built by the ICARUS collaboration. Its very precise imaging capabilities would mean very good background rejection, while maintaining high efficiency, as described by D. Cline. Because the backgrounds from neutral current events can be eliminated, and the signal efficiency remains high, the reach per kton of a liquid argon detector can be as much as 3 times that of other technologies. Therefore, one would require in principle a much smaller detector mass than those listed in the table above. Although using the 600 Ton module size of ICARUS would be prohibitively expensive even for a $6 \mathrm{kton}$ fiducial mass, $\mathrm{K}$. McDonald showed that for large enough single vessels, and large enough wire plane separation, this may become a very economical technology. Certainly the technology to make extremely large (order 100kton) cryogenic vessels is well-understood thanks 
to the natural gas companies, and the biggest challenge will likely be elsewhere.

For the NUMI Off Axis beamline, another detector technology being considered is fine-grained calorimetry, as described in a talk by A. Para. Fine grained calorimeters have a long history in the field of neutrino physics, and are well-understood and well-simulated. By making a low-Z calorimeter which was segmented every third of a radiation length, one could build a detector with reasonably high efficiencies, which would be able to reduce the neutral current background in the NUMI Off-Axis beamline to below that of the intrinsic $\nu_{e}$ contamination, which is itself about $0.4 \%$ under the peak energy. The challenge here will be to design a device that is economical to construct and realizes these large background reductions.

\subsection{Measurement Issues in Few GeV Beams}

As can be seen by the large number of proposals being considered, it is clear that one or more "neutrino superbeams" are likely to provide measurements before the advent of a neutrino factory. What's more, these neutrino superbeams are likely to be between a few hundred MeV region, such as the CERN SPL proposal, to the few (up to 6?) $\mathrm{GeV}$ region as in the BNL proposal. The experimental challenges associated with measurements with these beamlines are extremely different from those associated with those from a neutrino factory.

In the absence of oscillations, the number of events at a detector is simply the product of the neutrino flux times the cross section times the detector efficiency. Uncertainties in any of these can degrade the ability to use precise measurements of multiple transition probabilities to extract oscillation parameters, and in fact all of them may have complicated energy dependence, in particular in the sub to few $\mathrm{GeV}$ region. To address these issues, we convened a joint session with the neutrino scattering working group.

4.2.1. Cross Sections At very low energies, the cross-section is dominated by elastic or quasi-elastic processes which are few body reactions and relatively easy to model, at least in a free nucleon target. At very high energies, the cross-section is dominated by deep inelastic scattering, where complex multi-body final states can be effectively modeled with small corrections to a dominant picture of neutrino-quark scattering with an outgoing "jet" created by recoil hadronic system. However, the neutrino energies are on the order of the nucleon mass the second portion of the cross-section becomes dominated by intermediate resonant hadronic states with discrete masses, which complicate the picture significantly. In addition, the few body nature of the final state requires modeling exclusive reactions in order to correctly predict signal and background efficiencies.

Quasi-elastic cross-sections can be predicted very accurately, although the effects of final-state interactions in nuclei give 10-20\% uncertainties in observed rates. Deep inelastic cross-sections are known to a few percent in the high energy limit. However, in the "resonance" region the rates for exclusive final states typically have 50$100 \%$ uncertainties, and often differential distributions are completely unmeasured as discussed by Casper at this workshop, and as shown in Reference [4]. Bodek has been looking at a way to extend deep inelastic scattering predictions for cross sections to the resonance regime using global fits of many different data sets, and has shown that for charged lepton scattering, this treatment results in an accurate prediction of the mean cross section in the resonance region. By then using the data from charged 
leptons in the resonance region, it is hoped that this treatment can result in correct modeling of resonances in neutrino scattering at low energies [50].

4.2.2. Flux Predictions To first order, flux predictions will be made in these upcoming superbeam experiments by simply measuring the neutrino event distributions as a function of energy in a near detector and then by using a beamline simulation to extrapolate from near to far. However, this is complicated by the fact that the neutrino source is usually so close to the near detector that the source does not appear point-like. Also, if the neutrinos are from 3-body or non-pion decays, (i.e. the $\nu_{e}$ 's, which are from three-body decays of the muon and kaon) the near-far extrapolation is different.

Several different strategies for making flux predictions were discussed in this workshop. One very straightforward strategy in principle is to simply measure directly (or indirectly) the distributions of the secondaries that are creating the tertiary neutrinos in the first place. Kobayashi discussed the data from the pion monitor used by $\mathrm{K} 2 \mathrm{~K}$, which gives the angular distribution of pions at a given radius. The pion angular distribution, combined with the near detector measurements, gives a systematic uncertainty on the flux extrapolation to the far detector of $5 \%$ [51]. Coney discussed how MiniBooNE would be using muon counters located at the end of a small pipe set at a large angle with respect to the decay pipe at MiniBooNE. Most of the muons which were accepted at the end of that pipe are products of high angle kaon decays, and by measuring the energies of those muons, one could get a good constraint (estimated uncertainty goal of $10 \%$ ) on the ratio of pion to kaon decays in the beamline. This constraint is important for limiting the uncertainty in the kaon-induced $\nu_{e}$ background in MiniBooNE.

Another strategy for extrapolating from the near to the far detector was outlined by Szleper, using as an example the both the on and off-axis fluxes expected at the NUMI beamline. He considered, among other effects, the uncertainties in near to far extrapolation due to hadron production uncertainties. Because the bulk of the $\nu_{\mu}$ 's expected in both the near and far detectors are from pion decays, and the low energy $\nu_{e}$ 's from muon decays, where the muons were also from pion decays, Szleper showed how there is a straightforward correlation between what is measured in a near on-axis detector and far detectors, both on and off axis. The standard technique is simply to take the near detector energy spectrum and use the beamline simulation to create a "far over near ratio" for each energy bin, and then multiply by that ratio. The new technique developed by Szleper and Para, however, involves a "matrix approach" where for every neutrino event at the near on-axis detector there is a spectrum of events at the far detector, and those events are not be all reconstructed with the same energy as the event in the near detector [52]. For the $\nu_{\mu}$ 's coming from the focused pions, the two techniques result in similar hadron production uncertainties of 1-2\%, but for neutrinos for higher energy pions, those just underfocused, the differences are reduced from $15 \%$ (using the traditional approach) to 5\%. Para offered a wager of a bottle of good sake during the conference that if $\mathrm{K} 2 \mathrm{~K}$ were to use this technique their far detector flux would be predicted to $1 \%$, rather than to the $5 \%$ uncertainty currently assigned [51]. The error has yet to be reduced and no sake has yet changed hands.

Of course at a muon storage ring, the issues of flux prediction are quite different because the beam and decay kinematics as well known. As evidence of this point, Broncano showed that the $\mathcal{O}\left(\alpha_{E M}\right)$ corrections to the neutrino distributions were 
below the $0.1 \%$ level [53]. Therefore, it is likely that a neutrino factory will be in the enviable position of having the primary challenge to knowing the flux be knowledge of the phase space distribution and number of muons in the beam.

4.2.3. Near Detector Strategies Near Detectors play a very different role depending on whether or not one is discussing disappearance or appearance measurements. In the case of disappearance measurements, where it is assumed the oscillation probability will be large and the backgrounds to the signal small, the near detector is used to make an accurate prediction of the $\nu_{\mu}$ flux. In particular for a wide band neutrino beam, the energy dependence of both the flux and the detector response must be well understood. For appearance measurements, however, one wants to use a near detector to measure the backgrounds, and in particular how the backgrounds enter as a function of the neutrino energy, and the measured (visible) energy. For both kinds of measurements there are conflicting requirements for the near detector: one would like a detector that resembles the far detector as much as possible, but at the same time one would like a near detector that could make detailed studies of neutrino interactions in order to best model the far detector response.

One solution to this near detector problem is to have a suite of near detectors, as has been done very successfully by the K2K experiment, reported by Yoshida. While they have a $1 \mathrm{kT}$ Ton water Черенков device for a near to far detector ratio where the cross sections cancel, they can also compare events in the near water Черенков detector with those seen in their fine-grained scintillating fiber detector. The finegrained detector can see the recoil proton in the neutrino interaction, and K2K can use this to deduce the energy resolution and acceptance of their far detector. Furthermore, there is also a large muon chamber which can see the profile of the neutrino beam and verify that indeed the beam is pointing towards the far detector.

The success of this near detector suite is evidenced by the fact that very similar plans are in place for an off-axis near detector suite for the JHF to SuperKamiokande experiment, as described by Kajita. There will be near detectors at $280 \mathrm{~m}$ to study cross sections and verify the position of the neutrino beam, and a "less near" detector at $2 \mathrm{~km}$ consisting of a water Черенков device to make the most accurate far/near prediction [41, 54].

Because of the difference in energies and detector technologies, the MINOS experiment can use one near detector which will be used to not only measure the flux and event rates (using both quasielastic and non-quasielastic events, respectively), but which can also verify the direction of the neutrino beam (during special high energy runs). As described by Morfin, MINOS is also considering adding a speciallymodified cross section detector in front of the near detector, which can study neutrino interactions more precisely, and also can study the $\mathrm{Z}$ dependence of neutrino cross sections by having planes of various materials: carbon, steel, and tungsten [55].

For a NUMI off-axis experiment, however, one would like to study the detectorrelated backgrounds with an off-axis detector. This is because the on-axis beam spectrum is very different from the off-axis spectrum, and also because the visible energy in the background events is often far from the initial neutrino energy. One definite requirement for an off-axis experiment would be an off-axis near detector which mimics a far detector, but there have also been thoughts about also including a more fine-grained near detector, à la K2K. Bodek described a possible new detector for cross section studies that were relevant for a NUMI off-axis experiment, as well as a possible site for such a detector in the NUMI beamline [50]. 


\section{Conclusions}

From this workshop, it is clear that conventional neutrino beams will occupy the foreground of oscillation measurements until perhaps 2010. Then, a crucial decision will have to be made in choosing between hyper conventional experiments on one hand combining multi MegaWatt proton beams combined with MegaTon detectors, and neutrino factories on the other hand whose design should be mature by that time.

\section{References}

[1] J.W.F. Valle, in these proceedings.

[2] G.G. Ross, in these proceedings.

[3] T. Blazek, in these proceedings.

[4] Y. Shimizu, in these proceedings.

[5] N. Shimoyama, in these proceedings.

[6] M. Koike, in these proceedings.

[7] M. Fukugita and T. Yanagida, Phys. Lett. B 174 (1986) 45.

[8] T. Morozumi, in these proceedings.

[9] A. Ibarra, in these proceedings.

[10] Q. Shafi, in these proceedings.

[11] T. Kajita and Y. Totsuka, Rev. Mod. Phys. 73 (2001) 85.

[12] J. N. Bahcall, Phys. Rept. 333 (2000) 47.

[13] Z. Maki, M. Nakagawa and S. Sakata, Prog. Theor. Phys. 28 (1962) 870.

[14] B. Pontecorvo, Sov. Phys. JETP 26 (1968) 984 [Zh. Eksp. Teor. Fiz. 53 (1968) 1717].

[15] K. Hagiwara et al. [Particle Data Group Collaboration], Phys. Rev. D 66 (2002) 010001.

[16] M. Apollonio et al. [CHOOZ Collaboration], Phys. Lett. B 466 (1999) 415 arXiv:hep-ex/9907037.

[17] S. Choubey, in these proceedings.

[18] Q. R. Ahmad et al. [SNO Collaboration], Phys. Rev. Lett. 89 (2002) 011302 arXiv:nucl-ex/0204009.

[19] R. M. Barnett et al. [Particle Data Group Collaboration], Phys. Rev. D 54 (1996) 1.

[20] G. L. Fogli, E. Lisi, A. Marrone, D. Montanino and A. Palazzo, Phys. Rev. D 66 (2002) 053010 arXiv:hep-ph/0206162, and private communication.

[21] K. Kimura, in these proceedings.

[22] H. W. Zaglauer and K. H. Schwarzer, Z. Phys. C 40 (1988) 273.

[23] J. Burguet-Castell, M. B. Gavela, J. J. Gomez-Cadenas, P. Hernandez and O. Mena, Nucl. Phys. B 608 (2001) 301 arXiv:hep-ph/0103258.

[24] H. Minakata and H. Nunokawa, JHEP 0110 (2001) 001 arXiv:hep-ph/0108085.

[25] G. L. Fogli and E. Lisi, Phys. Rev. D 54 (1996) 3667 arXiv:hep-ph/9604415.

[26] V. Barger, D. Marfatia and K. Whisnant, Phys. Rev. D 65 (2002) 073023 arXiv:hep-ph/0112119.

[27] V. D. Barger, D. Marfatia and K. Whisnant, arXiv:hep-ph/0108090

[28] K. Whisnant, in these proceedings.

[29] O. Mena, in these proceedings.

[30] A. Donini, D. Meloni and P. Migliozzi, in these proceedings arXiv:hep-ph/0209240.

[31] P. Huber, in these proceedings arXiv:hep-ph/0210140.

[32] T. Ota, in these proceedings.

[33] B. Jacobsson, T. Ohlsson, H. Snellman and W. Winter, in these proceedings arXiv:hep-ph/0209147.

[34] C. Athanassopoulos et al. [LSND Collaboration], Phys. Rev. Lett. 77 (1996) 3082 arXiv:nucl-ex/9605003.

[35] H. Murayama and T. Yanagida, Phys. Lett. B 520 (2001) 263 arXiv:hep-ph/0010178.

[36] K.S. Babu, in these proceedings.

[37] Y.Y.Y. Wong, in these proceedings.

[38] J. Sato, in these proceedings.

[39] M. Campanelli, in these proceedings.

[40] W. J. Marciano, arXiv:hep-ph/0108181

[41] Y. Itow et al., arXiv:hep-ex/0106019 
[42] F. Dydak, Expression of interest in an experiment at the CNGS to measure $\theta_{13}$, http://home.cern.ch/dydak/oscexp.ps (April 2002).

[43] D. Beavis et al, BNL No. 52459, April 1995

[44] S. Kahn, in these proceedings, and D. Beavis et al., arXiv:hep-ex/0205040

[45] A. Ichikawa, in these proceedings.

[46] S. Kopp, in these proceedings, and D. Ayres, arXiv:hep-ex/0210005

[47] M. Mezzetto, in these proceedings.

[48] A. Cervera, A. Donini, M. B. Gavela, J. J. Gomez Cadenas, P. Hernandez, O. Mena and S. Rigolin, Nucl. Phys. B 579 (2000) 17 [Erratum-ibid. B 593 (2001) 731] arXiv:hep-ph/0002108

[49] H. J. Grabosch et al. [SKAT Collaboration], Z. Phys. C 41 (1989) 527.

[50] A. Bodek, in these proceedings.

[51] T. Kobayashi, in these proceedings.

[52] M. Szleper and A. Para, arXiv:hep-ex/0110001

[53] A. Broncano, in these proceedings.

[54] T. Kajita, in these proceedings.

[55] J. Morfin, in these proceedings. 